\title{
Antiobesity Activity of Liraglutide on High Fat Diet- Induced Obesity in Wistar Rats
}

\author{
Vinay Kumar* and Priynaka Gaur \\ Department of Pharmacology, India
}

Submission: March 21, 2017; Published: July 27, 2017

*Corresponding author: Vinay Kumar, Department of Pharmacology, KIET School of Pharmacy, India, Tel: 91-9711-060-878; Fax: 012-322-7978;

Email: vinaykumarpatel@gmail.com

\begin{abstract}
Liraglutide is a glucagon like peptide-1 (GLP-1) analogue. GLP-1 is a potent inhibitor of motility and gastric emptying and has also been shown to inhibit gastric acid secretion. The inhibition of gastric emptying leads to decreased food intake and reduced body weight. The aim of the present study was to investigate the antiobesity effect of the Liraglutide on high fat diet (HFD) induced obesity in Wistar rats. Obesity was induced by oral feeding of HFD for six weeks. The antiobesity effect of Liraglutide $(0.2 \mathrm{mg} / \mathrm{kg}$, i.p. for 14 days) in HFD fed rats was evaluated by the measurement of body mass index (BMI), body weight gain, food intake, hemodynamic changes (systolic, diastolic, mean blood pressure and heart rate), serum leptin, lipid profiles (triglycerides, total cholesterol, LDL-cholesterol, HDL-cholesterol), glucose. Organ (Liver) and visceral fat pad weights were measured. Liraglutide significantly reduced BMI, body weight gain, and mean blood pressure, serum leptin, lipids and glucose levels while it significantly increased the serum HDL-cholesterol, as compared to the HFD fed rats. The results of the present study suggest that a potential role of Liraglutide as a clinical tool for obesity treatment.
\end{abstract}

Keywords: Anti-obesity; High fat diet; Liraglutide; Leptin; Insulin

\section{Introduction}

Obesity has increased at an alarming rate in recent years and is now a worldwide public health problem [1]. It is one of the most common nutritional disorders in humans. Obesity can be defined as syndrome characterized by an increase in body fat stores, mainly due to an imbalance between energy intake and energy expenditure. Obesity results when energy intake exceeds energy expenditure. The global epidemic of obesity is rapidly evolving as one of the major global health issues as it is frequently associated with a number of diseases with high mortality and morbidity such as diabetes, cancer, arthritis, hypertension, stroke, and myocardial infarction [2]. It is generally accepted that the tremendous rise in the obesity prevalence across the globe is driven primarily by a combination of increased calorie intake and decreased physical activity, and strongly influenced by our genetic background [3]. Obesity is associated with substantial increases in morbidity, premature mortality, impaired quality of life and large healthcare costs [4]. The major co-morbidities include type 2 diabetes, metabolic syndrome, hypertension, dyslipidaemia, myocardial infarction, stroke, certain cancers, sleep, apnoea and osteoarthritis [5]. Easy access to high-calorie packaged foods, sedentary lifestyles and a predilection for gizmos have resulted in almost 70\% Indians in mega-cities such as Mumbai, Delhi, Bangalore or Chennai being overweight or obese, says a new multi-city survey. Diet and physical activity remain the cornerstones of therapy for obesity, although results have been disappointing. Obese patients who are able to lose weight by eating better and exercising generally regain the lost weight over time. The difficulty in maintaining long-term weight loss through behaviour modification has led to an increasing interest in other avenues of treatment, particularly pharmacotherapy. To date, only two medications-Sibutramine and Orlistat-have been approved for long-term use in the treatment of obesity and additional effective pharmacological treatments are needed [6].

Liraglutide is a glucagon-like peptide-1 receptor analogue, which is obtained by derivatising glucagon-like peptide- 1 with a fatty acid. The liraglutide is well tolerated, sustains weight loss over 2 years and improves cardiovascular risk factors [7]. Liraglutide suppress the food intake and body weight in rats [8]. Obesity management is a modern challenge because of the rapid evolution of unfavorable lifestyles and unfortunately there are no safe and effective treatments applicable to the large majority of obese/overweight people [9]. Therefore, the present study was designed to investigate the antiobesity effect of Liraglutide on high fat diet induced obesity in Wistar rats. 


\section{Materials and Methods}

\section{Animals}

The experimental study was carried out in male Wistar rats weighing 150-200g body weight and the experimental protocol was approved by Institutional Animal Ethics Committee (IAEC) of KIET School of Pharmacy, Ghaziabad (UP) (Registration number: 1099/07/CPCSEA, dated 27.07, 2007) as per the Committee for the Purpose of Control and Supervision of Experiments on Animals (CPCSEA) guidelines. The animals were kept in polypropylene cages (6 in each cage) under standard laboratory conditions (12 hrs. light and $12 \mathrm{hrs}$. dark :: day : night cycle) and had free access to commercial pellet diet (Pranav Agro Industries, New Delhi) and water ad libitum. The animal house temperature was maintained at $25 \pm 2{ }^{\circ} \mathrm{C}$ and relative humidity was also maintained at $50 \pm 15 \%$.

\section{Induction of obesity by feeding high fat diet}

Experimental obesity was induced by feeding of high fat diet consisting of the following materials (corn starch, casein, lard, cholesterol, sodium cholate, soybean oil, coconut oil, wheat bran, mineral mix, vitamin mix were added to $1 \mathrm{~kg}$ of rat chow diet) to rats for period of six weeks.

\section{Experimental design}

30 male Wistar rats were included in the present study. Animals were randomly allocated into five groups of six animals each and treated as follows: Normal Control Group: Rats fed with normal rat chow diet for 6 weeks; High Fat Diet Control Group: Rats fed with high fat diet for 6 weeks; Liraglutide Treated Group: Rats fed with high fat diet for 6 weeks+from $29^{\text {th }}$ day Liraglutide $(0.2 \mathrm{mg} / \mathrm{kg}$, i.p) for 2 weeks; Orlistat Treated Group: Rats fed with high fat diet for 6 weeks+from $29^{\text {th }}$ day Orlistat (10mg/kg, i.p) for 2 weeks; Perse Group: Rats fed with normal chow diet for 6 weeks+from $29^{\text {th }}$ day Liraglutide for 2 weeks.

Results

\section{Effect of liraglutide on BMI, food intake and water intake}

Table 1: Effect of liraglutide on body mass index, body weight, daily food intake and water intake.

\begin{tabular}{|c|c|c|c|c|}
\hline Groups & Body Mass Index $\left(\mathrm{kg} / \mathrm{m}^{2}\right)$ & Body Weight gain (gm) & Food Intake (gm/d/rat) & Water Intake (ml/d/rat) \\
\hline Normal control group & $4.26 \pm 1.05$ & $210 \pm 1.27$ & $14.5 \pm 0.67$ & $32 \pm 1.20$ \\
\hline High fat diet control group & $7.18 \pm 2.16^{\mathrm{a}}$ & $280 \pm 2.33^{\mathrm{a}}$ & $25.4 \pm 1.91^{\mathrm{a}}$ & $45 \pm 3.54^{\mathrm{a}}$ \\
\hline $\begin{array}{l}\text { High fat diet }+ \text { orlistat } \\
\text { treated group }\end{array}$ & $5.24 \pm 1.74^{b}$ & $172 \pm 2.89$ & $18.3 \pm 3.86^{b}$ & $39 \pm 2.87^{b}$ \\
\hline $\begin{array}{l}\text { High fat diet + liraglutide } \\
\text { treated group }\end{array}$ & $4.78 \pm 1.55^{b}$ & $157 \pm 3.23_{b}$ & $17.5 \pm 2.61^{b}$ & $40 \pm 1.78^{\mathrm{b}}$ \\
\hline Perse group (liraglutide) & $4.37 \pm 0.98^{c}$ & $207 \pm 1.68^{c}$ & $15.4 \pm 1.75^{c}$ & $34 \pm 0.33^{c}$ \\
\hline
\end{tabular}

All values were expressed as Mean \pm SEM, $(n=6)$; aP $<0.01$ as compared to the Normal Control Group; ${ }^{\mathrm{b}}<0.05$ as compared to the high fat diet Group; ' $\mathrm{P}>0.05$ as compared to Normal Control Group.

The mean BMI, food intake and water intake were significantly $(\mathrm{p}<0.01)$ increased in high fat diet fed group as compared to the normal control group. Liraglutide $(0.2 \mathrm{mg} / \mathrm{kg}$, i.p) and Orlistat $(10 \mathrm{mg} / \mathrm{kg}$, i.p) treatment significantly $(\mathrm{p}<0.05)$

\section{Measurement of anthropometric parameters}

Body mass index (BMI $\left.\mathrm{kg} / \mathrm{m}^{2}\right)$, daily food intake $(\mathrm{g} / \mathrm{d})$ and daily water intake $(\mathrm{ml} / \mathrm{d})$ were measured.

\section{Measurement of hemodynamic parameters}

Hemodynamic parameters (systolic, diastolic and mean arterial blood pressure) were measurement by non-invasive blood pressure recorder using rat tail-cuff method (AD Instrument, Australia).

\section{Biochemical estimations in serum}

Blood was collected from the retro-orbital plexus of the both groups of overnight fasted rats using microcapillary tubes on $29^{\text {th }}$ day. Serum was separated by centrifugation $(4000 \mathrm{rpm}$, $10 \mathrm{~min}$ ) and transferred to Eppendorf tubes. The concentrations high density lipoprotein-cholesterol (Reckon Diagnostics Pvt. Ltd., Baroda, Gujarat, India), glucose, total cholesterol (TC) and triglycerides (TGs) (all the three from Span diagnostics Ltd., Surat, Gujarat, India), in serum were measured with commercial kits. The concentration of leptin in the serum was measured with rat leptin ELISA kit (BioVendor, Brno, Czech Republic).

\section{Determination of Liver Weight and Visceral Fat Pad Weights}

On the final day of experiment the rats were fasted overnight and sacrificed by cervical dislocation and then liver and visceral fat pads (epididymal, perirenal and mesentric) were removed, washed with normal saline and weighed [10].

\section{Statistical analysis}

All values were expressed as Mean $\otimes$ Standard Error of Mean (S.E.M). Statistical analysis was performed using InStat 3Software. Groups of data were compared with the analysis of variance (ANOVA) followed by Dunnett's test. Values were considered statistically significant when $\mathrm{p}<0.05$. 


\section{Current Research in Diabetes \& Obesity Journal}

\section{Effect of liraglutide on hemodynamic parameters}

Table 2: Effect of liraglutide on heart rate, systolic, diastolic, mean blood pressure in wistar rats.

\begin{tabular}{|c|c|c|c|c|}
\hline Groups & Heart Rate & Systolic BP(mmHg) & Diastolic BP(mmHg) & Mean BP(mmHg) \\
\hline Normal control group & $421.83 \pm 3.67$ & $127.83 \pm 2.86$ & $96 \pm 4.65$ & $106.16 \pm 5.00$ \\
\hline High fat diet control group & $539.20 \pm 5.38^{\mathrm{a}}$ & $165.16 \pm 3.58^{\mathrm{a}}$ & $119 \pm 4.56^{\mathrm{a}}$ & $135.33 \pm 6.09^{a}$ \\
\hline Orlistat treated group & $381.33 \pm 4.37^{b}$ & $118.25 \pm 4.26^{\mathrm{b}}$ & $87.5 \pm 3.39^{b}$ & $97.5 \pm 3.56^{b}$ \\
\hline Liraglutide treated group & $452 \pm 2.09^{\mathrm{b}}$ & $133 \pm 1.69^{\mathrm{b}}$ & $95.83 \pm 3.51^{\mathrm{b}}$ & $106.66 \pm 2.67^{b}$ \\
\hline Perse group (liraglutide) & $420 \pm 5.77^{c}$ & $123.45 \pm 6.12^{c}$ & $95 \pm 4.36^{c}$ & $104.32 \pm 4.83_{c}$ \\
\hline
\end{tabular}

All values were expressed as Mean \pm SEM, $(n=6)$; ${ }^{a}<<0.01$ as compared to the Normal Control Group; ${ }^{b} P<0.05$ as compared to the high Fat Diet Group; ${ }^{c} \mathrm{P}>0.05$ as compared to Normal Control Group.

All hemodynamic parameters (systolic, diastolic, mean arterial BP and heart rate) were significantly increased $(\mathrm{p}<0.01)$ in high fat diet treated group as compared to the normal control group. All hemodynamic parameters were significantly decreased $(\mathrm{p}<0.01)$ in Liraglutide and Orlistat treated groups as compared to HFD treated group (Table 2).

\section{Effect of liraglutide on serum leptin and glucose levels}

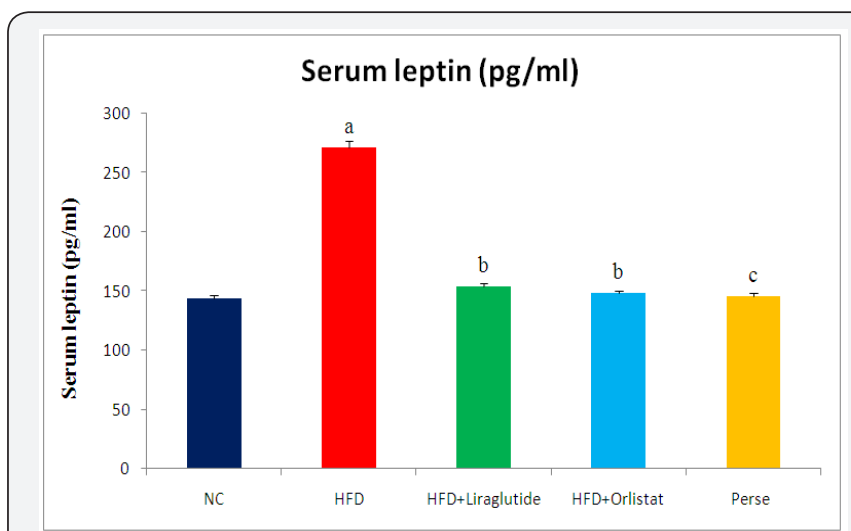

Figure 1: Effect of liraglutide on serum leptin levels in wistar rats.

All values were expressed as Mean \pm SEM, $(n=6)$; ${ }^{a} P<0.01$ as compared to the Normal Control Group; ${ }^{b} P<0.05$ as compared to the high Fat Diet Group; ' $\mathrm{P}>0.05$ as compared to Normal Control Group.

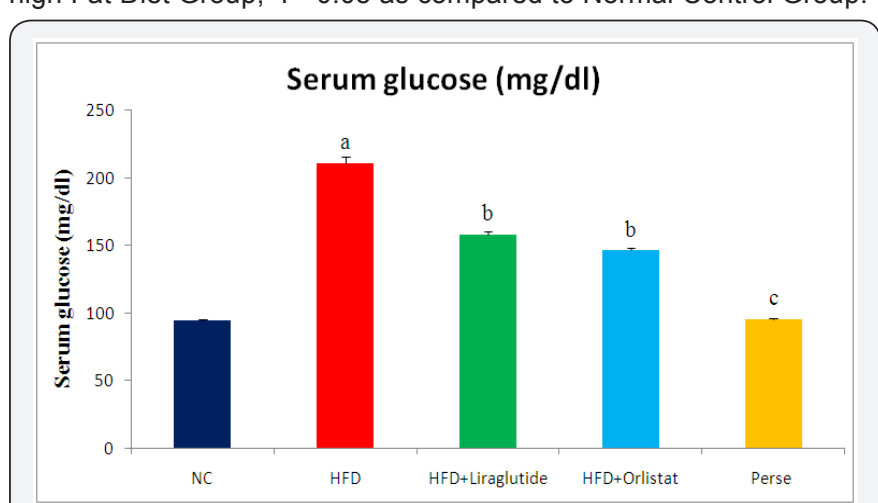

Figure 2: Effect of liraglutide on serum glucose levels in wistar rats.

All values were expressed as Mean \pm SEM, $(n=6)$; ${ }^{a} P<0.01$ as compared to the Normal Control Group; ${ }^{\mathrm{b}} \mathrm{P}<0.05$ as compared to the high Fat Diet Group; ${ }^{c}>0.05$ as compared to Normal Control Group.

Figure $1 \& 2$ shows that Liraglutide treatment serum leptin and glucose levels were significantly $(p<0.01)$ increased in HFD treated rats as compared to the normal control rats. Serum leptin and glucose levels were significantly decreased in Liraglutide and Orlistat treated groups as compared to the HFD fed group.

\section{Effect of liraglutide on serum lipid profile levels}

HFD treated group showed significant $(p<0.01)$ increased levels TC, TGs, low density lipoprotein-cholesterol (LDL-C) and very low density lipoprotein-cholesterol (VLDL-C) and while HDL-C levels significantly $(\mathrm{p}<0.01)$ decreased as compared to the normal control group. All these levels were significantly reversed in Liraglutide and Orlistat treated groups as compared to the HFD fed group (Table 3).

\section{Effect of liraglutide on liver weight and visceral fat pad weights}

Liver weight and visceral fat pad weights (mesenteric, perirenal and epididymal) were significantly increased in HFD fed group as compared to the normal control group. The visceral fat pad weights of perirenal fat, mesenteric and epididymal fat in the HFD + Liraglutide and HFD + orlistat groups were significantly $(\mathrm{p}<0 \bullet 01)$ decreased as compared with those of the HFD group (Table 4).

\section{Discussion}

Obesity is a medical condition in which excess body fat has been accumulated mainly due to sedentary life styles, lack of exercise and intake of energy rich high fat diet. The global prevalence of obesity is increasing rapidly among adults as well as among children and is associated with serious mortalities including a high incidence of type 2 diabetes, hyperlipidemia, hypercholesterolemia, fatty liver, cardiovascular diseases, osteroarthritis as well as an increased risk of many forms of cancer [11]. The currently available treatment options are not potent enough to control obesity permanently besides they produce side effects. Hence, there is a great demand for safer and long term effective drugs to treat this global epidemic problem. Hence, in the present work the anti-obesity activity of Liraglutide on high fat diet induced obesity in Wistar was investigated by analyzing the BMI, body weight, organ and fat pad weight and blood biochemical profiles. Dietary fat is calorically dense and extremely palatable. It is easily over consumed because it can cause less satiety than carbohydrate and protein [12]. Therefore, a HFD can lead to hyperphagia, weight gain, and 


\section{Current Research in Diabetes \& Obesity Journal}

increased adiposity. The feeding of HFD for 4 weeks produced a significant increase in body weight, total fat pad weight, basal/ fasting plasma glucose, insulin, basal triglyceride (TG) and total cholesterol (TC) levels in male rats [13]. In present study, there was significant increase in body mass index (BMI) and body weight gain in rats fed with high fat diet as compared to the normal control rats. BMI is a simple index of weight-for-height that is commonly used to classify underweight, overweight and obesity in adults [14]. This study corroborate with the findings of Altunkaynak [15] who reported that BMI was significantly increased in rats with high fat diet fed for 8 weeks as compared to the control group The increased body weight found in HFD rats might be due to the consumption of a diet rich in energy in the form of saturated fats (lard) and its deposition in various body fat pads and decreased energy expenditure.

Liraglutide $(0.2 \mathrm{mg} / \mathrm{kg}$, i.p) and Orlistat $(10 \mathrm{mg} / \mathrm{kg}$, i.p) treatment significantly $(\mathrm{p}<0.01)$ decreased the BMI and body weight gain as compared to the HFD treated group. Further, our results showed a significant $(\mathrm{p}<0.05)$ decrease in food intake and water intake by administration of Liraglutide $(0.2 \mathrm{mg} / \mathrm{kg}$, i.p). After 6 weeks feeding with HFD, hemodynamic parameters were significantly $(\mathrm{p}<0.01)$ elevated in HFD fed rats as compared to normal healthy control rats. Activation of the sympathetic nervous system contributes to blood pressure (BP) elevation in high-fat diet-induced obesity [16]. When HFD fed rats were treated with Liraglutide $(0.2 \mathrm{mg} / \mathrm{kg}$, i.p), there were significant $(p<0.01)$ decrease in hemodynamic changes.

In the present study, serum glucose and leptin levels were increased in the HFD group as compared to the normal control group, while glucose levels were significantly decreased by Orlistat and Liraglutide treatment. Diet-induced obesity dysregulated glucose homeostasis and causes hyperglycemia [17]. This is consistent with previous study that feeding of HFD for a period of 4 weeks produced a significant increase in plasma glucose levels. Fried et al. [18] indicated that basal levels of leptin are known to be strongly positively correlated with body fat on a HFD. A fat-enriched diet is regarded as an important factor in the development of cardiac diseases because it leads to the development of hyperlipidemia, atherosclerosis, and abnormal lipid metabolism [19]. The data of present study clearly showed that feeding of the HFD increased the concentrations of serum TC, LDL- C, VLDL-C, TGs in experimental rats. Lavie \& Milani [20] indicated that obesity adversely affects plasma lipids, especially by increasing TC, LDL- C, VLDL-C, TGs and decreasing the level of HDL-cholesterol. The levels of TGs, TC, LDL- C, VLDL-C were significantly $(\mathrm{p}<0.01)$ decreased by Liraglutide $(0.2 \mathrm{mg} / \mathrm{kg}$, i.p) and Orlistat treatment ( $10 \mathrm{mg} / \mathrm{kg}$, i.p) treatment groups. Organ's weight (liver) and visceral fat pad weights (perirenal, mesentric fat and epididymal fat) were significantly increased in rats fed with HFD. HFD produces adiposity i.e. deposition of fat on the adipose tissues. Liver weight and visceral fats were significantly decreased by Liraglutide.

\section{Conclusion}

The possible explanation for an anti-obesity effect of Liraglutide via suppression of dyslipidaemia, leptin, insulin, gastric emptying and reduction of appetite in obese rats fed with HFD. The results of the present study provide initial evidence that Liraglutide could be useful intervention for the treatment of obesity.

\section{References}

1. Friedman JM (2000) Obesity in the new millennium. Nature 404(6778): 632-634.

2. Haslam DW, James WP (2005) Obesity. Lancet 366(9492): 1197-1209.

3. Shalaby HMA, Tawfek NS, Abo El Hussein BK, Abd El Ghany (2014) The assessment of some biochemical and immunological effects by amphetamine and Orlistat on obesity in rats. Food and Public Health 4(4): 185-192.

4. Kopelman PG (2000) Obesity as a medical problem. Nature 404(6778): 635-643.

5. Flegal KM, Graubard BL, Williamson DF, Gail MH (2005) Excess deaths associated with underweight, overweight and obesity. JAMA 293(15): 1861-1867.

6. Bray GA, Ryan DH (2012) Medical therapy for the patient with obesity. Circulation 125(13): 1695-1703.

7. Astrup A, Carraro R, Finer N, Harper A, Kunesova M (2012) Safety, tolerability and sustained weight loss over 2 years with the once-daily human GLP-1 analog, Liraglutide. Int J Obes (Lond) 36(6): 843-854.

8. Hayes MR, Kanoski SE, Alhadeff AL, Grill HJ (2011) Comparative effects of the long-acting GLP-1 receptor ligands, Liraglutide and exendin-4, on food intake and body weight suppression in rats. Obesity (Silver Spring) 19(7): 1342-1349.

9. Pagotto U, Vanuzzo D, Vicennati V, Pasquali R (2008) Pharmacological therapy of obesity. G Ital Cardiol (Rome) 9(4 Suppl 1): 83S-93S.

10. Kaur G, Kulkarni SK (2000) Antiobesity effect of a polyherbal formulation, OB-200G in female rats fed on cafeteria and atherogenic diets. Ind J Pharmacol 32(5): 294-299.

11. Yilmaz A, Suleyman H, Umudum Z, Sahin YN (2002) The effect of adrenalectomy on leptin levels and some metabolic parameters in rats with diet-induced obesity. Biol Pharma Bull 25(5): 580-583.

12. Rolls BJ, Hammer VA (1995) Fat, carbohydrate, and the regulation of energy intake. Am J Clin Nutr 62(5 Suppl): 1086S-1095S.

13. Srinivasan K, Patole PS, Kaul CL, Ramarao P (2004) Methods Find Exp Clin Pharmacol 26(5): 327-333.

14. (WHO) World Health Organization Expert Committee. Physical Status: The use and interpretation of anthropometry, Geneva, Swizerland.

15. Altunkaynak Z (2005) Effects of high fat diet induced obesity on female rat livers. Eur J Gen Med 2(3): 100-109.

16. Iwashita S, Tanida M, Terui N, Ootsuka Y, Shu M, et al. (2002) Direct measurement of renal sympathetic nervous activity in high-fat dietrelated hypertensive rats. Life Sci 71(5): 537-546.

17. Chang S, Graham B, Yakubu F, Lin D, Peters JC, et al. (1990) Metabolic differences between obesity-prone and obesity-resistant rats. Am J Physiol 259(6 Pt 2): R1103-R1110.

18. Fried SK, Ricci MR, Russell CD, Laferrere B (2000) Regulation of leptin production in humans. J Nutr 130(12): 3127S-3131S. 


\section{Current Research in Diabetes \& Obesity Journal}

19. Onody AM, Csonka C, Giricz Z, Ferdinandy P (2003) Hyperlipidemia induced by a cholesterol-rich diet leads to enhanced peroxidative formation in rat hearts. Cardiovasc Res 58(3): 663-670.
20. Lavie CJ, Milani RV (2003) Obesity and cardiovascular disease: Hippocrates paradox? J Am Coll Cardiol 42(4): 677-679.

Your next submission with Juniper Publishers will reach you the below assets

- Quality Editorial service

- Swift Peer Review

- Reprints availability

- E-prints Service

- Manuscript Podcast for convenient understanding

- Global attainment for your research

- Manuscript accessibility in different formats (Pdf, E-pub, Full Text, Audio)

- Unceasing customer service

Track the below URL for one-step submission https://juniperpublishers.com/online-submission.php 